Vitaliy Korendiy

Department of Mechanics and Automation Engineering, Lviv Polytechnic National University, 12, S. Bandery Str., Lviv, Ukraine, E-mail: vitalii.m.korendii@lpnu.ua

\title{
STRUCTURAL AND KINEMATIC SYNTHESIS OF THE 1-DOF EIGHT- BAR WALKING MECHANISM WITH REVOLUTE KINEMATIC PAIRS
}

\author{
Received: July 12, 2017 / Revised: September 16, 2017 / Accepted: December 26, 2017
}

(C) Korendiy V., 2017

\begin{abstract}
Problem statement. The use of existing and the most widespread drives (wheeled and caterpillar one) is sometimes limited by complicated operational conditions while moving on rough terrain. The mentioned drives require a relatively flat surface to be operated effectively. A rocky or a hilly terrain imposes the demand of the use of alternative types of drives, in particular, walking ones. Purpose. In this paper, there will be proposed and analysed one of the possible structures of the walking mechanism for mobile robotic system to be used on rough terrain. Methodology. While carrying out the investigations, the structural and kinematic synthesis of the eight-bar hinge-lever walking mechanism has been performed using the well-known methods of the Theory of Machines and Mechanisms, in particular, the method of closed vector loops. In order to conduct experimental investigations and simulation of the mechanism motion, the applied software SolidWorks and MapleSim has been used. Findings (results). The structure and geometrical parameters of the eight-bar walking mechanism have been synthesized with the aim to ensure the required trajectory and kinematic characteristics of the supporting foot motion. Originality (novelty). The analytical dependencies describing the trajectory of each hinge of the analysed walking mechanism have been derived and the kinematic optimization synthesis problem has been solved. This allowed to substantiate of the mechanism's geometrical parameters and to analyse its kinematic characteristics. Practical value. The proposed structure of the walking mechanism can be effectively used in various mobile robotic systems and in transporting and technological machines in order to ensure the possibility of their use on rough terrain where there is no ability to use wheeled and caterpillar drives. Scopes of further investigations. While carrying out further research, it is necessary to analyse the influence of the weight coefficients on the solution of the optimization problem, as well as to take into account the necessity of changing the step length and the foot lifting height during the process of walking according to the surface obstacles sizes.
\end{abstract}

Keywords: walking mechanism; structural analysis; kinematic analysis; synthesis; optimization; objective function; geometrical parameters; trajectory; speed; acceleration.

\section{Introduction}

Nowadays, there exist a wide range of various drives of transporting and technological machines: wheeled, crawler-type (caterpillar), walking (stepping), rotor-and-auger-type, with air (pneumatic) or electromagnetic cushion etc. Two of them, namely, wheeled and crawler-type ones, have found wide application. Three other drives, namely, air-cushion, rotor-and-auger-type and walking ones, are in a varying degree used in individual models of transporting vehicles. At the same time, the drive based on the electromagnetic cushion is used only by the railway transport.

The walking drive has the widest range of various designs and has found the application both in heavy technological machines (excavators, reclamation and irrigation machines, logging (timber harvesting) machines etc.) and in modern structures of androids, exoskeletons and walkers (walking movers) [1]-[4]. The application of walking drives allows significant reducing of loads acting upon the supporting surface. At the same time, the mass of the technological machine may reach several hundred tons. 


\section{Problem Statement}

The development of simple, reliable and, at the same time, relatively accurate walking mechanisms of mobile robotic systems and technological machines provides the necessity of solving two major problems: structural and kinematic synthesis of the mechanism with simultaneous optimization of its parameters in accordance with the prescribed motion characteristics of the supporting foot and machine body [4]-[7]. One of the simplest and most widespread variants of designing the supporting link of the walking mechanism is presented in Fig. 1 [4]-[16]. In the present paper, we will try to synthesize the 1-DOF eight-bar mechanism able to implement the straight-line path of the supporting foot motion and the stability of its horizontal speed during the whole phase of its contact with the supporting surface with the simultaneous minimization of durations of the foot lifting, returning and lowering phases. These conditions are stipulated by the necessity of ensuring the horizontal position of the machine body during its motion, the necessity of prevention of the supporting feet slipping during their simultaneous contact with the supporting surface, as well as with the aim of ensuring the stability of the equilibrium (static) position and motion of the walking machine [4]-[16].

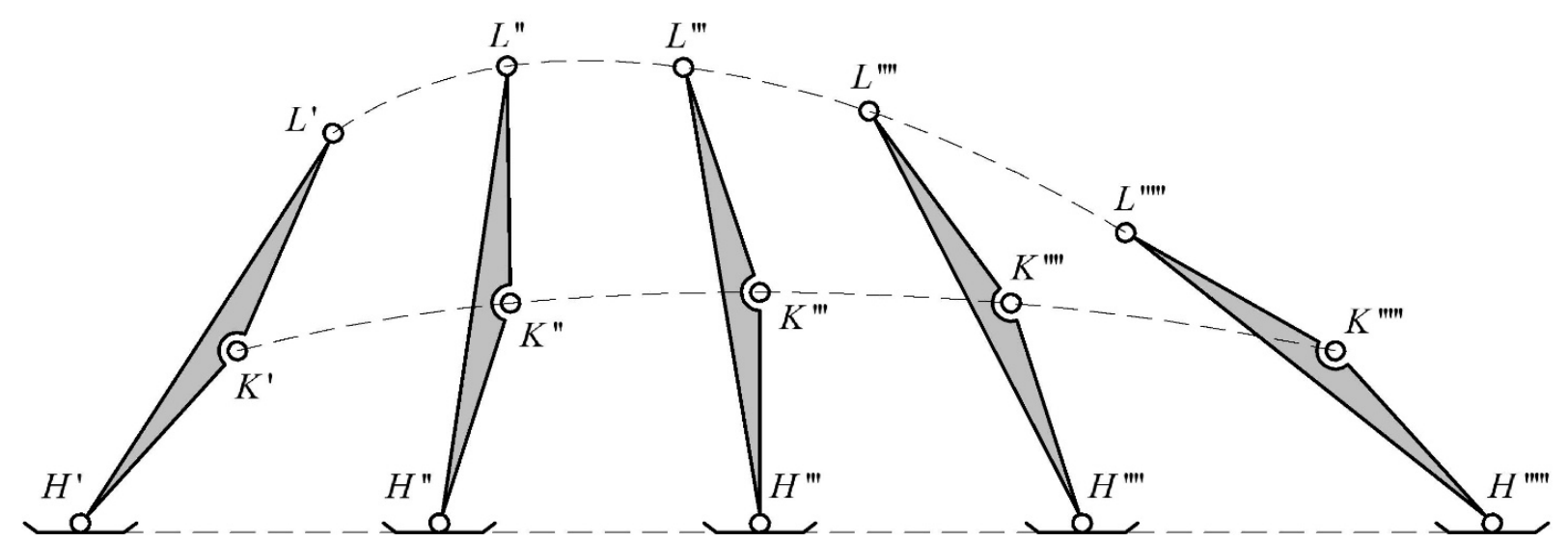

Fig. 1. The scheme of motion implementation of the output link of the walking mechanism

As it may be seen in Fig. 1, in order to ensure the horizontal trajectory of the supporting foot (hinge $H$ ), it is necessary to provide circular motion paths of hinges $L$ and $K$. With a help of these hinges, the supporting lever is connected to the intermediate links of the walking mechanism. Using only two mechanisms of the first class, it is impossible to implement these paths [17]. That's why in further investigations, there will be considered the possibilities of usage of two-link structural groups (structural groups of the second class) and one mechanism of the first class being used as the driving member.

\section{Review of Modern Information Sources on the Subject of the Paper}

During the last several years, the particular interest among researchers and engineers is caused by walking (stepping) machines based on rigid lever systems, in particular, the drives based on cyclic mechanisms [4]-[16]. Such drives guarantee a prescribed trajectory of the supporting foot motion and are characterized by the fact that they use a ready mechanical transformer or a new mechanism, which is synthesized according to the chosen trajectory of the supporting foot. The advantages of cyclic walking movers also include the simplicity and reliability of the legs and drives structures, because usually such mechanisms require only one degree of freedom [4]-[16].

Despite the considerable number of advantages of hinge-lever walking mechanisms, at present we do not observe their widespread usage in transport and technological machines. Of course, there are separate pilot and experimental models (prototypes) of equipment, but they have not yet been implemented in serial production [1]-[3]. This is due to some disadvantages of cyclic hinge-lever mechanisms. Firstly, this is the complexity of avoiding the vertical movements of the body of the walking machine during the motion, which is caused by the non-translational motion trajectory of the foot in the zone of its contact with the supporting surface [5]. Secondly, it is impossible to ensure a steady horizontal foot speed in the 
zone of its contact with the supporting surface, which causes its slipping [13], [15]. Thirdly, it is the complexity of ensuring the stability of the equilibrium position and the motion of the walking machine with a minimum number of supporting legs (2-4) [4], [16].

To some extent, the mentioned disadvantages may be fixed by increasing the number of supporting foots [1]-[3]; by increasing the number of links in the walking mechanism [6]-[12]; by involvement of additional driving degrees of freedom [4], which allow to change the geometric parameters of separate links; by the use of controllable drives that allow continuous changing of the driving link speed [3]. These methods allow rational limiting the duration of the supporting foot contact phase, more accurate ensuring the prescribed trajectory of its motion and the stability of its horizontal speed during the contact phase of, as well as ensuring the stability of equilibrium position and motion of the walking machine.

\section{Objectives and Problems of Research}

The aim of this work is to substantiate the geometric parameters of an eight-link hinge-lever walking drive in order to provide a prescribed motion trajectory of the supporting foot and a stability of its velocity in the phase of contact with a supporting surface because of the necessity to negotiate the obstacles along the walking path and to limit the processes of the foot slipping in the phase of contact with a supporting surface.

\section{Structural Synthesis of the Walking Mechanism}

Let us adopt the Assur groups of $2^{\text {nd }}$ class $2^{\text {nd }}$ order $1^{\text {st }}$ type [17] as driven and intermediate structural groups of the walking mechanism (Fig. 2, $a-c$ ). Thus, we obtain a crank-connecting rod-rocker mechanism only with single-motion revolute (turning) kinematic pairs (Fig. 3). The supporting lever 1 of the walking mechanism is hingedly (pivotally) joined with the connecting rod 2 and the rocker 5 . The connecting rod 2 is hingedly (pivotally) connected with the rocker 3, to which the motion is transmitted from the crank 7 through the connecting rod 4 . The rocker 5 is driven by the crank 7 with a help of the connecting rod 6 . The links 3, 5 and 7 are connected to the fixed (stationary) member 0 (machine body) with a help of hinges $B, C$ and $O$.

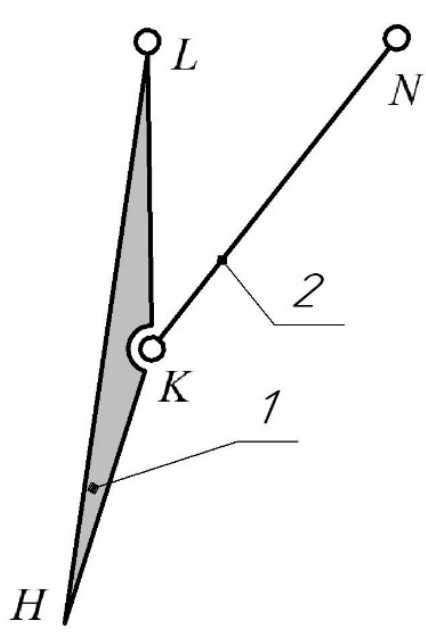

$a$

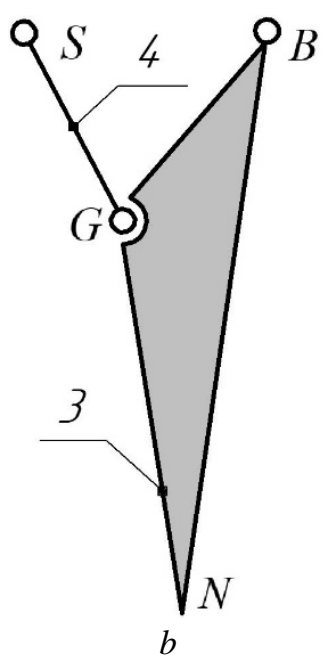

$b$

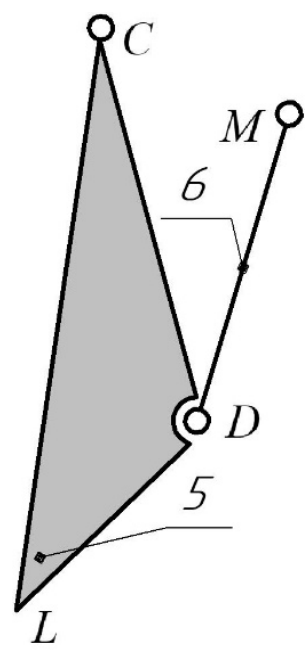

$c$

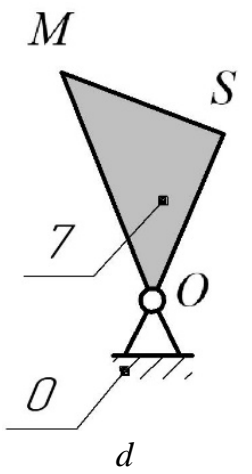

Fig. 2. Structure groups, which form the walking mechanism

In the obtained walking mechanism (Fig. 3), the point $H$ should move along the straight line on the certain trajectory section, which is called the supporting phase or the pahse of foot interaction with supporting surface. The other section of the point $H$ trajectory (its curved part) is called the phase of the foot transportation. During the process of the mechanism moving, the connecting rods 1, 2, 4, 6 are in the planar (plane-parallel) motion; the rockers 3 and 5 are in oscillatory (reciprocating) motion; the crank 7 is in rotary motion. Thus, the mechanism (Fig. 3) consists of the seven movable links $(n=7)$ and ten kinematic pairs of the $5^{\text {th }}$ class (single-motion revolute (turning) kinematic pairs, $\left.p_{5}=10\right): K(1,2)$, 
$N(2,3), G(3,4), L(1,5), D(5,6), S(4,7), M(6,7), B(3,0), C(5,0), O(7,0)$. There are no higher kinematic couples in the mechanism, that is $p_{4}=0$. The degree of freedom in the hinge $H$ is uncontrolled and serves for self-adapting of the foot according to the supporting surface irregularities. That's why, the total number of degrees of freedom of the eight-bar walking mechanism may be calculated with a help of the formula derived by Chebyshov [17]:

$$
W=3 \cdot n-2 \cdot p_{5}-p_{4}=3 \cdot 7-2 \cdot 10-0=1 .
$$

Therefore, the walking mechanism has one degree of freedom and thus one input link and one independent coordinate, which exactly determines the position of all other parts of the mechanism. Let us accept the crank 7 with rotation axis $O$ (Fig. 4) as an input link of the walking mechanism. In that case, the angle $\varphi$, which defines the deflection of the crank 7 (OS) from the horizontal axis directed from the point $O$ to the right (Fig. 4), may be concerned as the generalized coordinate for further kinematic analysis of the mechanism. The positive direction of the angle $\varphi$ reference is counterclockwise.

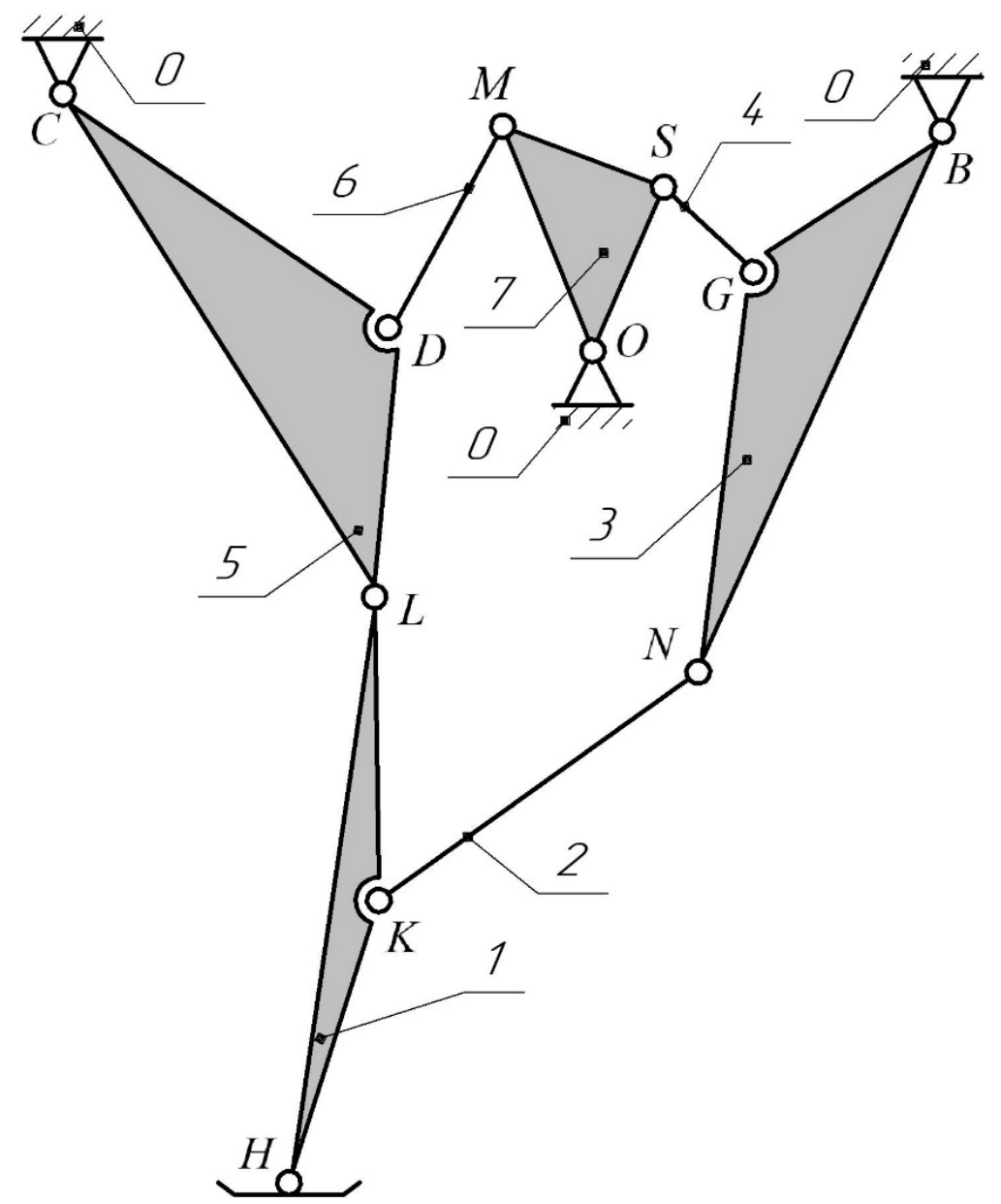

Fig. 3. Structure diagram of the walking mechanism

With the aim of choosing the rational method of further kinematics analysis of the walking mechanism (Fig. 3), let us expand it into the groups of Assur. Let's separate the loops which consist of the following parts: links 1 and 2 with three kinematic pairs (joints) $L, N, K$ (Fig. 2,a); links 3 and 4 with three kinematic pairs (joints) $G, S, B$ (Fig. 2, b); links 5 and 6 with three kinematic pairs (joints) $C, D$, $M$ (Fig. 2,c). After this we receive one input link 7 (Fig. 2, $d$ ), which is characterized by the degree of freedom $W=1$ (the mechanism of the first class). Therefore, the mentioned loops may be concerned as the 
structural groups of the second class second order first type [17]. Let us write down the structure formula of the eight-bar walking mechanism:

$$
\begin{array}{lllll}
\text { I (fixed pillar 0, crank 7) } & \text { Z } & \text { II (coupler 4, rocker 3) } & \text { ] } & \text { II (coupler 1, coupler 2) }
\end{array}
$$

In order to analyse the walking mechanism, the equations of motion of the foot joint are needed. This means that the coordinates of the foot joint presented as a function of the input link rotation, the lengths of each link of the mechanism, and the angle of the base link are needed [3]. Based on the results of structure analysis, let us accept the method of closed vector loops for further kinematics analysis of the walking mechanism. This method was developed by V. A. Zinoviev and consists in expanding the mechanism into separate closed vector loops and deducing vector equations of closeness of each loop [17].

\section{Derivation of Kinematic Parameters of the Supporting Foot Motion}

Let us consider the eight-bar hinge-lever walking mechanism with one degree of freedom, which is driven by the crank $O S$ (Fig. 4). Let us place the centre of the Cartesian coordinate system in the point $O$; let us direct the $O x$ axis horizontally to the right; let us direct the $O y$ axis vertically upwards. Let us adopt the angle $\varphi$ of the crank $O S$ deflection from the right horizontal position as the generalized coordinate. Let us adopt the positive direction of the crank motion as anticlockwise one.

In accordance to the method of closed vector loops, the motion trajectories of the points $G$ and $D$ may be presented as follows:

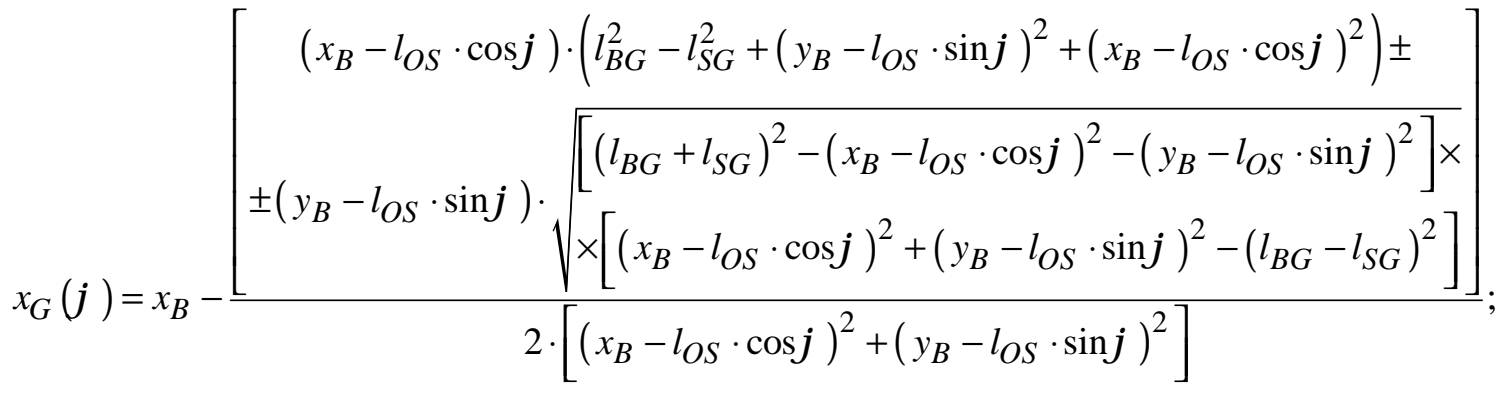

$$
\begin{aligned}
& y_{G}(\varphi)=l_{O S} \cdot \sin \varphi-
\end{aligned}
$$

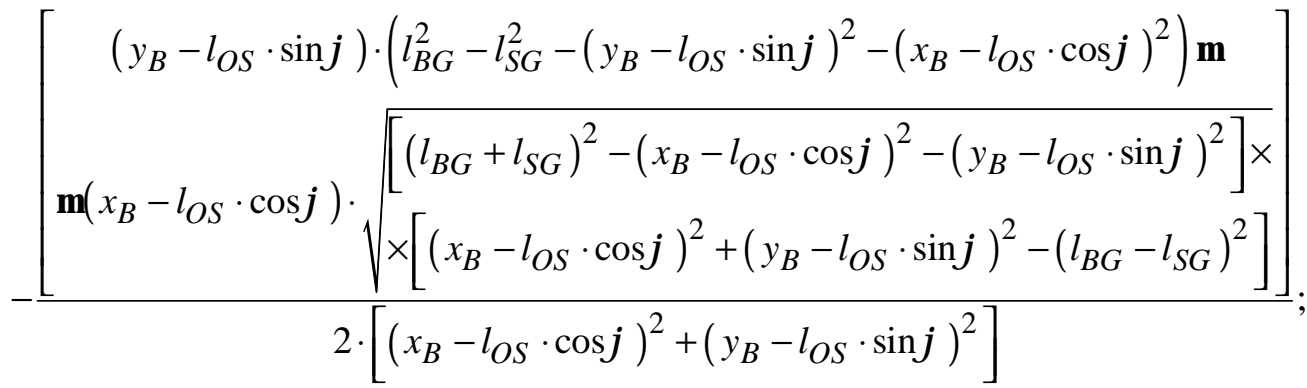

$$
\begin{aligned}
& x_{D}(\varphi)=x_{C}+\frac{\left[\begin{array}{c}
\left(x_{C}-l_{O M} \cdot \cos (\varphi+\alpha)\right) \times \\
\times\left(l_{C D}^{2}-l_{M D}^{2}+\left(y_{C}-l_{O M} \cdot \sin (\varphi+\alpha)\right)^{2}+\left(x_{C}-l_{O M} \cdot \cos (\varphi+\alpha)\right)^{2}\right) \pm \\
\pm\left(y_{C}-l_{O M} \cdot \sin (\varphi+\alpha)\right) \times
\end{array}\right.}{\times \sqrt{\left[\begin{array}{l}
\left.\left(l_{C D}+l_{M D}\right)^{2}-\left(x_{C}-l_{O M} \cdot \cos (\varphi+\alpha)\right)^{2}-\left(y_{C}-l_{O M} \cdot \sin (\varphi+\alpha)\right)^{2}\right] \times \\
\times\left[\left(x_{C}-l_{O M} \cdot \cos (\varphi+\alpha)\right)^{2}+\left(y_{C}-l_{O M} \cdot \sin (\varphi+\alpha)\right)^{2}-\left(l_{C D}-l_{M D}\right)^{2}\right]
\end{array}\right]}}
\end{aligned}
$$




$$
\begin{aligned}
& y_{D}(\varphi)=l_{O M} \cdot \sin (\varphi+\alpha)- \\
& \left(y_{C}-l_{O M} \cdot \sin (\varphi+\alpha)\right) \times \\
& \times\left(l_{C D}^{2}-l_{M D}^{2}-\left(y_{C}-l_{O M} \cdot \sin (\varphi+\alpha)\right)^{2}-\left(x_{C}-l_{O M} \cdot \cos (\varphi+\alpha)\right)^{2}\right) \mathrm{m} \\
& m\left(x_{C}-l_{O M} \cdot \cos (\varphi+\alpha)\right) \times \\
& \frac{\times \sqrt{\left[\begin{array}{l}
\left.\left(l_{C D}+l_{M D}\right)^{2}-\left(x_{C}-l_{O M} \cdot \cos (\varphi+\alpha)\right)^{2}-\left(y_{C}-l_{O M} \cdot \sin (\varphi+\alpha)\right)^{2}\right] \times \\
\times\left[\left(x_{C}-l_{O M} \cdot \cos (\varphi+\alpha)\right)^{2}+\left(y_{C}-l_{O M} \cdot \sin (\varphi+\alpha)\right)^{2}-\left(l_{C D}-l_{M D}\right)^{2}\right]
\end{array}\right]}}{2 \cdot\left[\left(x_{C}-l_{O M} \cdot \cos (\varphi+\alpha)\right)^{2}+\left(y_{C}-l_{O M} \cdot \sin (\varphi+\alpha)\right)^{2}\right]},
\end{aligned}
$$

where $l_{O S}, l_{B G}, l_{S G}, l_{O M}, l_{C D}, l_{M D}$ are the lengths of the corresponding links of the walking mechanism (Fig. 4); $x_{B}, y_{B}, x_{C}, y_{C}$ are the coordinates of the fixed (stationar) cylindrical hinges of the rockers connections; $\alpha$ is the constant angle between the levers $O S$ and $O M$.

In accordance to the method of closed vector loops, the motion trajectories of the points $N$ and $L$ may be presented as follows:

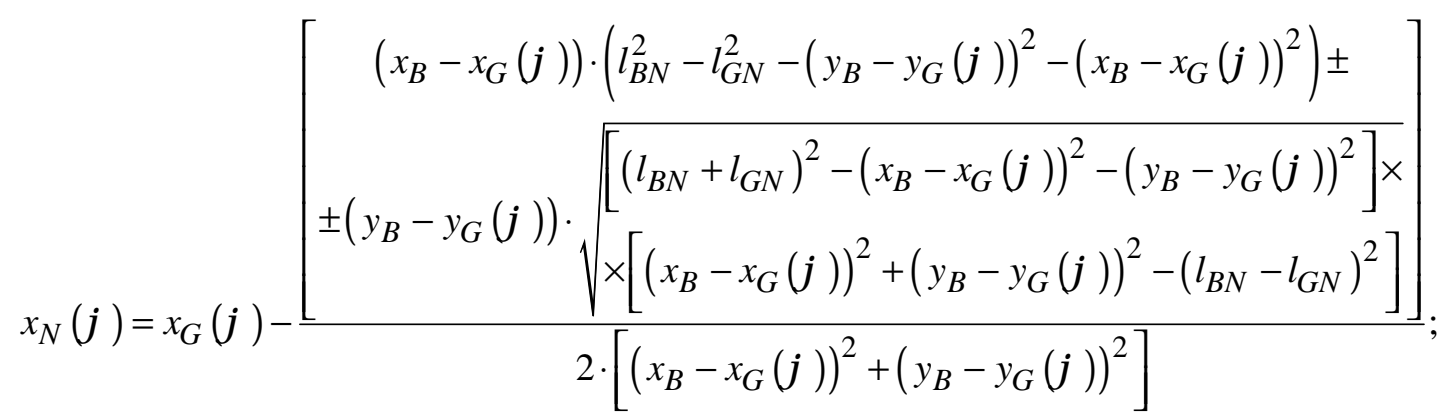

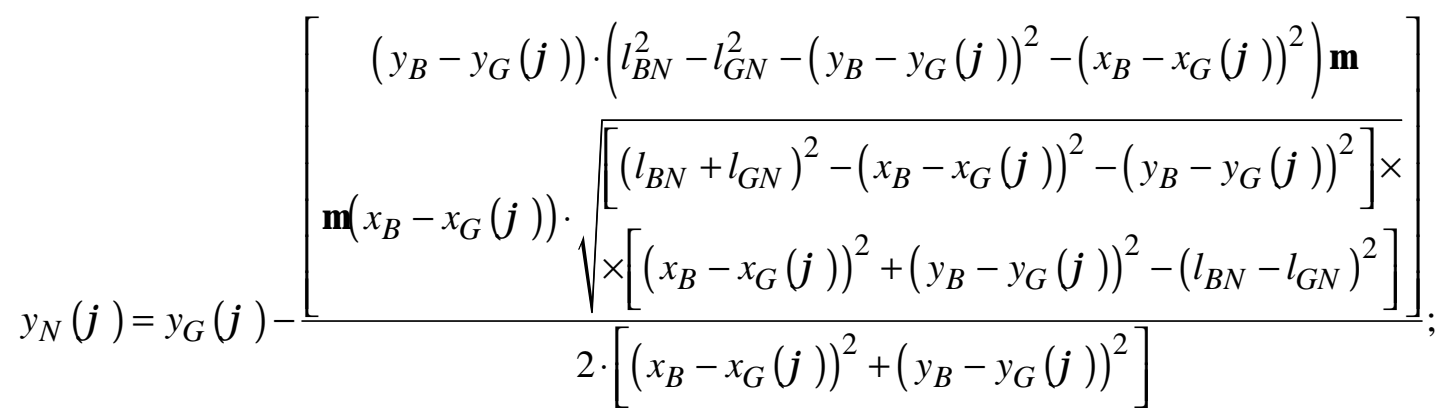

$$
x_{L}(\varphi)=x_{D}(\varphi)+\frac{\left[\begin{array}{c}
\left(x_{C}-x_{D}(\varphi)\right) \cdot\left(l_{C L}^{2}-l_{D L}^{2}-\left(y_{C}-y_{D}(\varphi)\right)^{2}-\left(x_{C}-x_{D}(\varphi)\right)^{2}\right) \pm \\
\pm\left(y_{C}-y_{D}(\varphi)\right) \cdot \sqrt{\left[\left(l_{C L}+l_{D L}\right)^{2}-\left(x_{C}-x_{D}(\varphi)\right)^{2}-\left(y_{C}-y_{D}(\varphi)\right)^{2}\right] \times} \times\left[\left(x_{C}-x_{D}(\varphi)\right)^{2}+\left(y_{C}-y_{D}(\varphi)\right)^{2}-\left(l_{C L}-l_{D L}\right)^{2}\right]
\end{array} ;\right.}{2 \cdot\left[\left(x_{C}-x_{D}(\varphi)\right)^{2}+\left(y_{C}-y_{D}(\varphi)\right)^{2}\right]}
$$




$$
y_{L}(\varphi)=y_{D}(\varphi)-\frac{\left[\begin{array}{c}
\left(y_{C}-y_{D}(\varphi)\right) \cdot\left(l_{C L}^{2}-l_{D L}^{2}-\left(y_{C}-y_{D}(\varphi)\right)^{2}-\left(x_{C}-x_{D}(\varphi)\right)^{2}\right) \mathrm{m} \\
\mathrm{m}\left(x_{C}-x_{D}(\varphi)\right) \cdot \sqrt{\left[\left(l_{C L}+l_{D L}\right)^{2}-\left(x_{C}-x_{D}(\varphi)\right)^{2}-\left(y_{C}-y_{D}(\varphi)\right)^{2}\right] \times} \times\left[\left(x_{C}-x_{D}(\varphi)\right)^{2}+\left(y_{C}-y_{D}(\varphi)\right)^{2}-\left(l_{C L}-l_{D L}\right)^{2}\right]
\end{array}\right]}{2 \cdot\left[\left(x_{C}-x_{D}(\varphi)\right)^{2}+\left(y_{C}-y_{D}(\varphi)\right)^{2}\right]},
$$

where $l_{B N}, l_{G N}, l_{C L}, l_{D L}$ are the lengths of the corresponding links of the walking mechanism (Fig. 4); $x_{G}(\varphi), y_{G}(\varphi), x_{D}(\varphi), y_{D}(\varphi)$ are the dependencies of the coordinates of corresponding movable cylindrical hinges $G$ and $D$, which may be defined with a help of formulas (3).

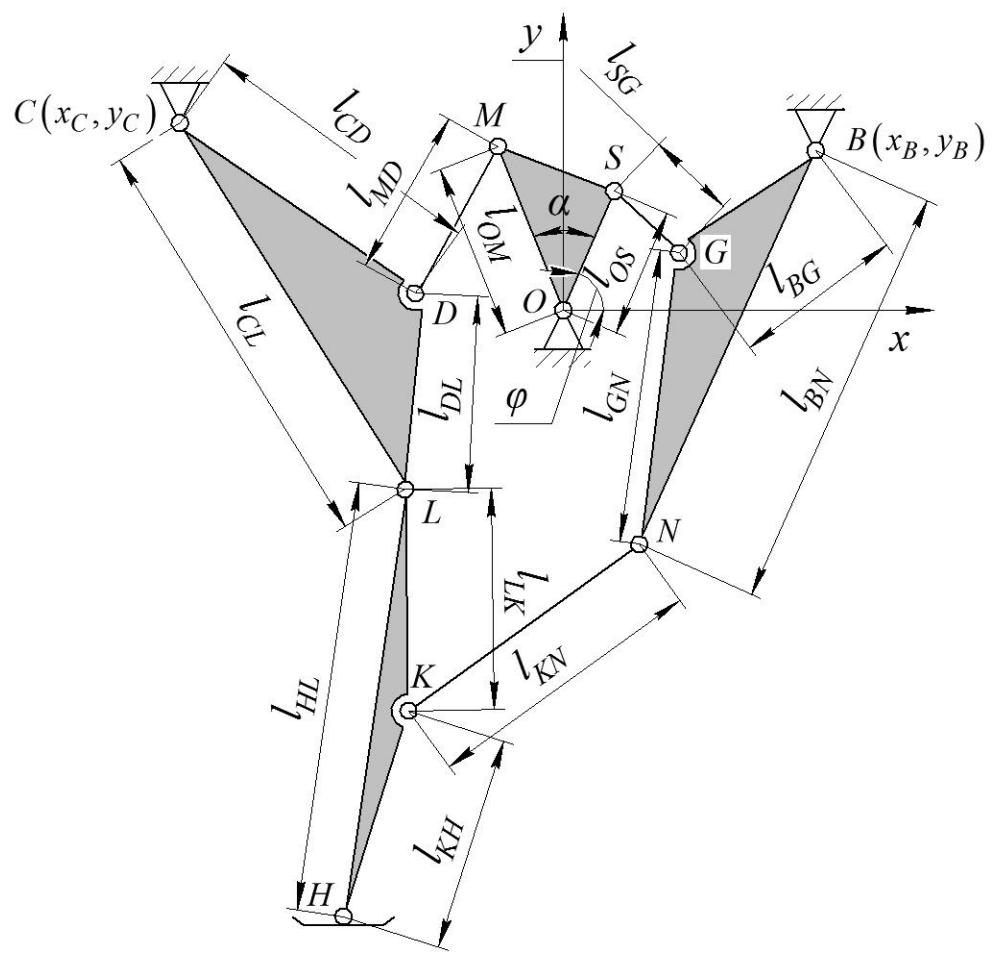

Fig. 4. Kinematic diagram of the walking mechanism

In accordance to the method of closed vector loops, the motion trajectories of the points $K$ and $H$ may be presented as follows:

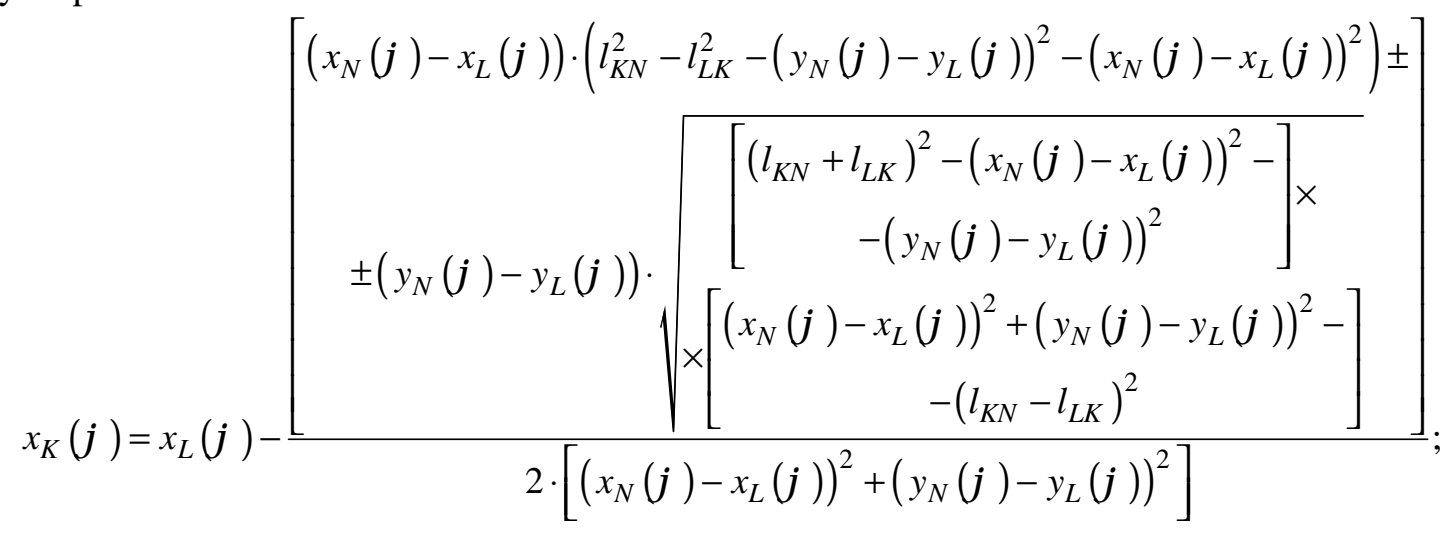




$$
\begin{aligned}
& y_{K}(\varphi)=y_{L}(\varphi)-\frac{\left[\begin{array}{c}
\left(y_{N}(\varphi)-y_{L}(\varphi)\right) \cdot\left(l_{K N}^{2}-l_{L K}^{2}-\left(y_{N}(\varphi)-y_{L}(\varphi)\right)^{2}-\left(x_{N}(\varphi)-x_{L}(\varphi)\right)^{2}\right) \mathrm{m} \\
m\left(x_{N}(\varphi)-x_{L}(\varphi)\right) \cdot\left[\begin{array}{c}
{\left[\left(l_{L K}+l_{K N}\right)^{2}-\left(x_{N}(\varphi)-x_{L}(\varphi)\right)^{2}-\right] \times} \\
-\left(y_{N}(\varphi)-y_{L}(\varphi)\right)^{2}
\end{array}\right] \\
2 \cdot\left[\left(x_{N}(\varphi)-x_{L}(\varphi)\right)^{2}+\left(y_{N}(\varphi)-y_{L}(\varphi)\right)^{2}\right]
\end{array}\right]}{-\left[\begin{array}{c}
\left(x_{N}(\varphi)-x_{L}(\varphi)\right)^{2}+\left(y_{N}(\varphi)-y_{L}(\varphi)\right)^{2}- \\
-\left(l_{K N}-l_{L K}\right)^{2}
\end{array}\right]},
\end{aligned}
$$

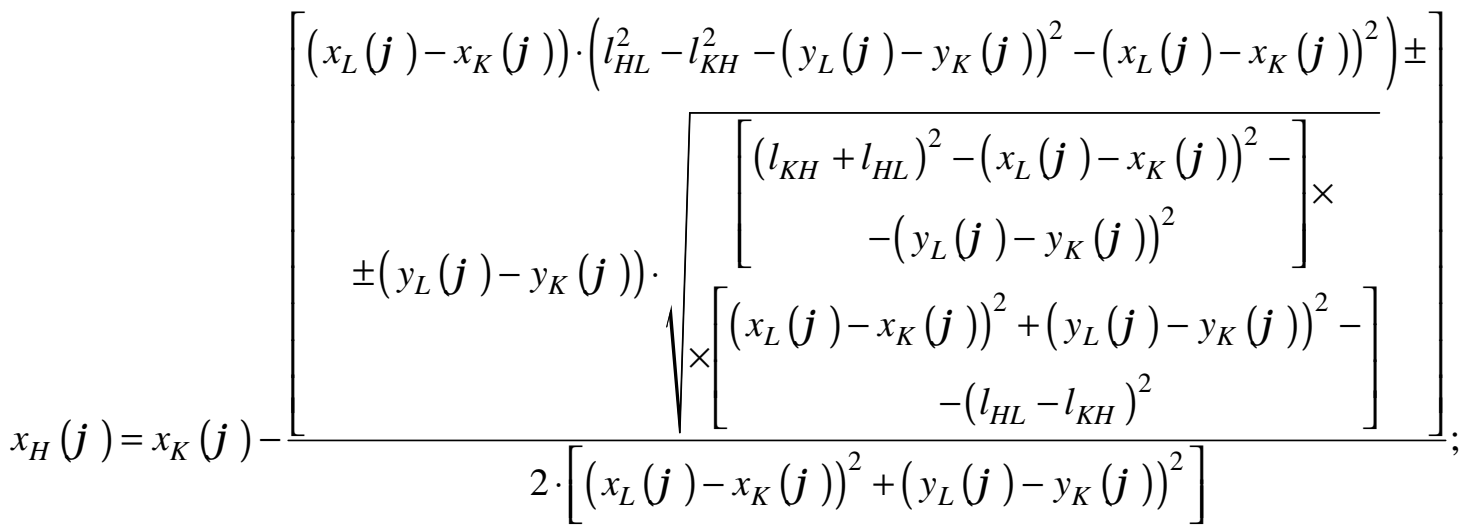

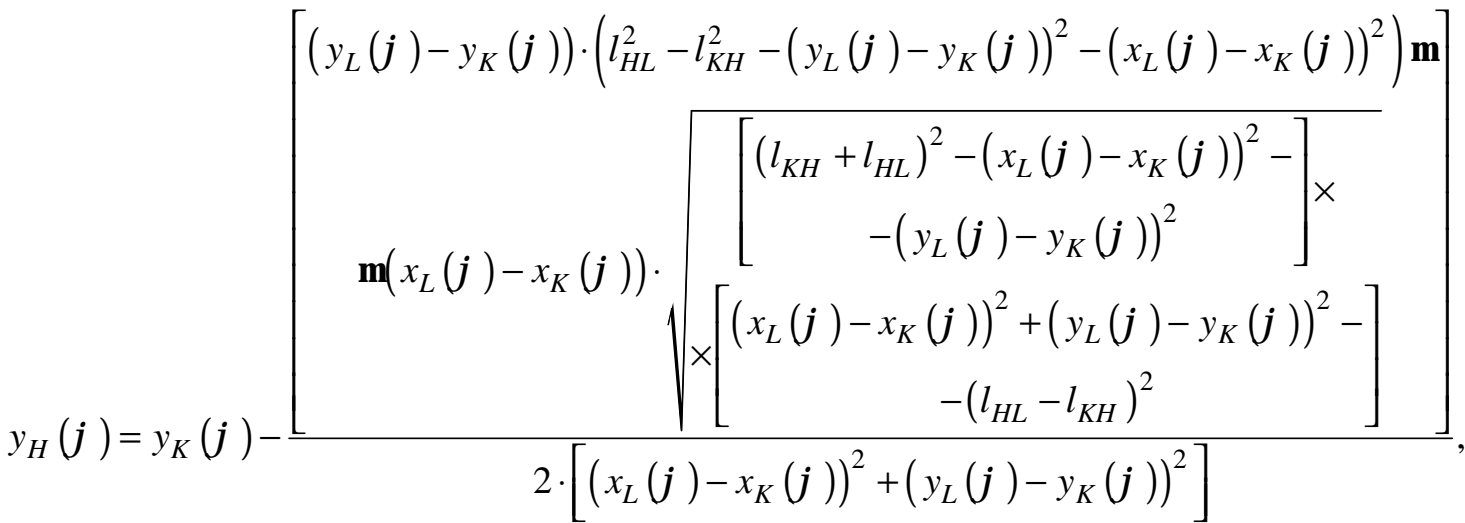

where $l_{K N}, l_{L K}, l_{H L}, l_{K H}$ are the lengths of the corresponding links of the walking mechanism (Fig. 4); $x_{N}(\varphi), y_{N}(\varphi), x_{L}(\varphi), y_{L}(\varphi)$ are the dependencies of the coordinates of corresponding movable cylindrical hinges $N$ and $L$, which may be defined with a help of formulas (4).

Thus, the motion trajectory of the supporting point $H$ of the synthesized eight-bar walking mechanism may be described in the parametric form by the dependencies (5).

\section{Kinematic Synthesis of the Walking Mechanism}

Let us consider the problem of kinematic synthesis of the hinge-lever walking mechanism, which consists in substantiation of geometrical parameters of the links in order to ensure the prescribed kinematic characteristics of the mechanism operation (for example, the positions of links, the motion trajectories of certain points, their speeds and accelerations etc.). Let us adopt the trajectory of the supporting point $H$ of the walking mechanism as the basic (fundamental) input parameter (Fig. 4). The output parameters of the kinematic synthesis will be the geometrical sizes of the mechanism links: $l_{O S}, l_{O M}, l_{S G}, l_{M D}, l_{C D}, l_{B G}$, $l_{C L}, l_{D L}, l_{G N}, l_{B N}, l_{L K}, l_{K N}, l_{H L}, l_{K H}, x_{B}, y_{B}, x_{C}, y_{C}, \alpha$. 
The objective (target) function may be presented in the form of the sum of absolute deviations of the point $H$ trajectory from the prescribed path in $N$ given positions [5], [18]:

$$
\Delta_{1}=\sum_{i=1}^{N} \sqrt{\left(x_{H_{i}}-x_{i}\right)^{2}+\left(y_{H_{i}}-y_{i}\right)^{2}},
$$

where $x_{H i}, y_{H i}$ are the coordinates of the point in $i$-th position of the mechanism, which may be determined by the formulas (5); $x_{i}, y_{i}$ are the coordinates of the point on the prescribed curve (path) in the same $i$-th position of the mechanism.

Thus, in order to solve the problem of kinematic synthesis of the crank-connecting rod-rocker walking mechanism, it is necessary to minimize the function (6) for as many as possible positions of the mechanism. For example, let us define 6 positions of the supporting point $H$ on the theoretical trajectory of its motion (Fig. 5, Table 1) taking into account the necessary step length $(700 \mathrm{~mm})$ and the height of the foot lifting $(200 \mathrm{~mm})$. Five points define the straight-line trajectory of the foot motion during the phase of its contact with the supporting surface and the six one defines the maximal height of the foot lifting in the transportation phase.

Table 1

The input parameters of the synthesis

(the coordinates of 6 positions of the supporting point $H$ )

\begin{tabular}{|c|c|c|c|c|c|c|}
\hline$\varphi_{i},{ }^{\circ}$ & 0 & 90 & 180 & 240 & 300 & 360 \\
\hline$x_{i}, \mathrm{~mm}$ & 150 & -200 & -550 & -320 & -90 & 150 \\
\hline$y_{i}, \mathrm{~mm}$ & -1750 & -1550 & -1750 & -1750 & -1750 & -1750 \\
\hline
\end{tabular}

During the process of the walking drives designing, it is necessary to take into account the fact that simultaneous contact of several foots with the supporting surface requires the stabilization of horizontal components of speeds of corresponding supporting points. The large difference between the horizontal speeds of several feet in the contact phase may cause the slipping of the walking drive, changing the machine motion trajectory, or even the failure (breakdown) of some elements. The presence of large horizontal accelerations in the phase of the foot contact with the supporting surface also causes the increasing of energy losses due to the periodic speeding-up and slowing-down of the machine body during its motion.

Thus, the additional synthesis restriction consists in the necessity to ensure the stability of horizontal speed (or horizontal zero-acceleration) of the supporting point $H$ in the phase of the foot contact with the supporting surface [5]:

$$
\left.\frac{\partial^{2} x_{H}(\varphi)}{\partial \varphi^{2}}\right|_{\varphi=180^{\circ} \ldots 360^{\circ}}=0
$$

In such a case, the expression of the objective function (6) will be complemented by one more component:

$$
\Delta_{2}=\sum_{i=1}^{P}\left|\frac{\partial^{2} x_{H}\left(\varphi_{i}\right)}{\partial \varphi_{i}^{2}}\right|,
$$

and the objective function will have the following form:

$$
\Delta=k_{1} \cdot \Delta_{1}+k_{2} \cdot \Delta_{2}=k_{1} \cdot \sum_{i=1}^{N} \sqrt{\left(x_{H_{i}}-x_{i}\right)^{2}+\left(y_{H_{i}}-y_{i}\right)^{2}}+k_{2} \cdot \sum_{i=1}^{P}\left|\frac{\partial^{2} x_{H}\left(\varphi_{i}\right)}{\partial \varphi_{i}^{2}}\right|,
$$

where $P$ is the total number of the prescribed positions of the supporting point $H$, which defines the straight-line trajectory of its motion in the phase of contact with the supporting surface; $k_{1}, k_{2}$ are the 
weight coefficients of each of the optimization criterions, which satisfies the following conditions: $k_{1}=\overline{0.1}, k_{2}=\overline{0.1}, k_{1}+k_{2}=1$.

Substituting by terns the coordinates of the supporting point $H$ presented in Table 1 into the equation (9) and using the function of the local minimum defining $\operatorname{Minimize}\left(\Delta, l_{O S}, l_{O M}, l_{S G}, \alpha, \ldots\right)$ in the MathCAD software [17], we obtain the following values of unknown parameters for $k_{1}=k_{2}=0.5$ : $l_{O S}=l_{O M}=89.5 \mathrm{~mm}, \alpha=0^{\circ}, l_{S G}=397.7 \mathrm{~mm}, l_{B G}=330.4 \mathrm{~mm}, l_{B N}=730 \mathrm{~mm}, l_{G N}=401.3 \mathrm{~mm}$, $l_{K N}=1167.2 \mathrm{~mm}, \quad l_{M D}=302.5 \mathrm{~mm}, \quad l_{C D}=550.9 \mathrm{~mm}, \quad l_{D L}=394.9 \mathrm{~mm}, \quad l_{C L}=793.7 \mathrm{~mm}$, $l_{L K}=975.2 \mathrm{~mm}, \quad l_{H L}=1427.8 \mathrm{~mm}, \quad l_{K H}=452.9 \mathrm{~mm}, \quad x_{B}=274.2 \mathrm{~mm}, \quad y_{B}=325.9 \mathrm{~mm}$, $x_{C}=-139.2 \mathrm{~mm}, y_{C}=405.9 \mathrm{~mm}$.

The trajectory of the supporting foot motion of the crank-connecting rod-rocker walking mechanism with the geometrical parameters presented above, which is constructed on the basis of analytical dependencies (3)-(5), is presented in Fig. 5.

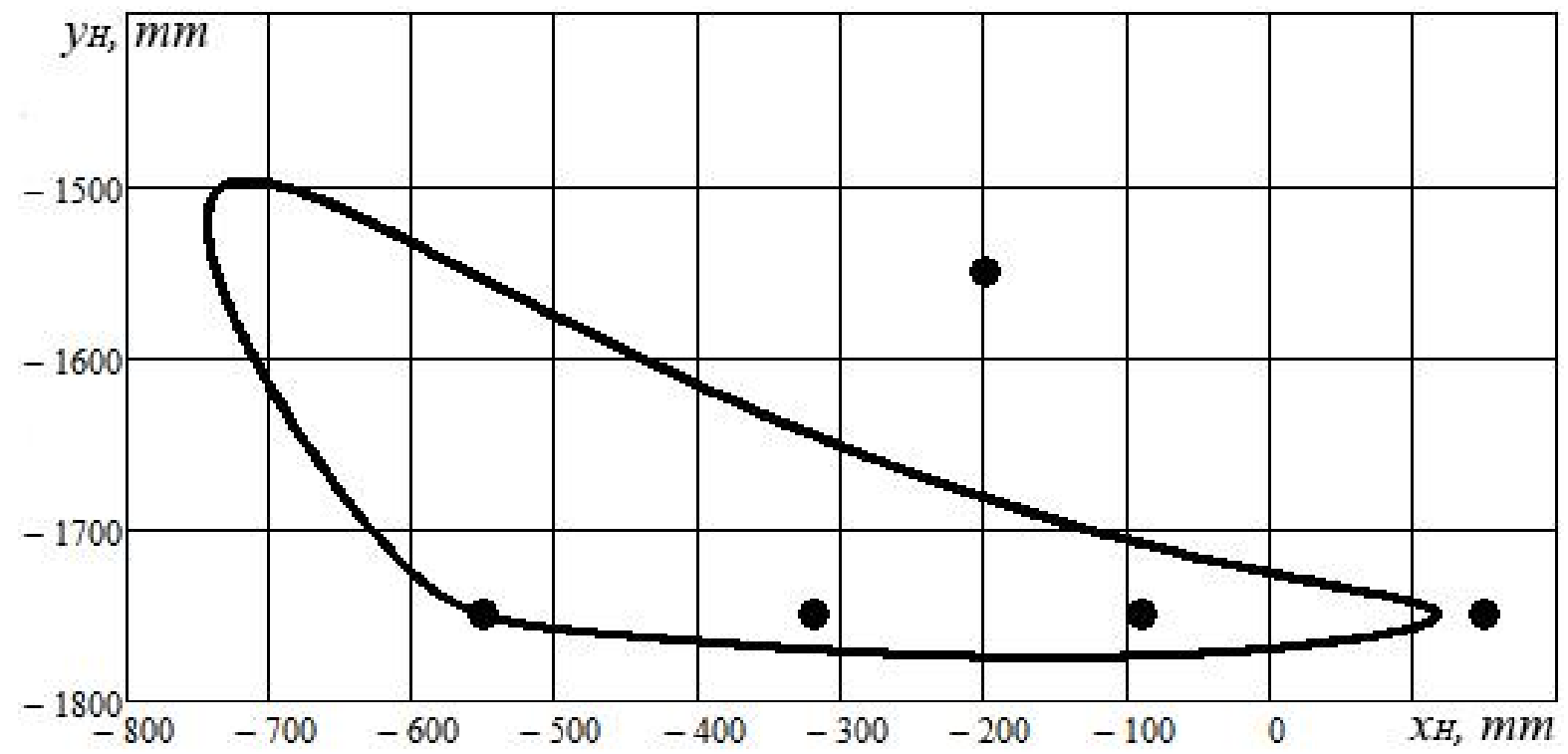

Fig. 5. The coordinates of the 6 prescribed positions of the supporting point and the synthesized trajectory of its motion

Considering the trajectory of the supporting foot motion of the eight-bar walking hinge-lever mechanism (Fig. 5), we may conclude that the deflection of the machine body (i.e., the distance between the top and bottom end positions of the supporting point $H$ in the phase of contact with the supporting surface) does not exceed $30 \mathrm{~mm}$ and ensures the limitation of the energy losses due to the periodic liftingup and lowering-down of the machine body during its motion. Herewith, the maximal height of the foot lifting equals $250 \mathrm{~mm}$, and the step length is $700 \mathrm{~mm}$, i.e., all the input conditions of the kinematic synthesis are almost satisfied.

It is necessary to mention, that the accuracy of the unknown parameters determination depends on the number of points prescribed on the theoretical foot motion trajectory and on the accuracy of defining the initial estimates of unknown parameters. For this, it is necessary to decrease the number of unknown parameters and to impose the restrictions on the lengths, displacements and speeds of some elements of the mechanism [18].

Substituting the synthesized parameters of the walking mechanism into the equations (3)-(5) and taking the corresponding derivatives with respect to rotation angle of the driving crank, let us construct the graphical dependencies (plots) of horizontal displacement, speed and acceleration of the supporting point 
$H$ on the angle $\varphi$ assuming that $\varphi=\omega \cdot t$ and the angular velocity of the driving crank rotation is equal $\omega=1 \mathrm{rad} / \mathrm{s}$ (Fig. 6).

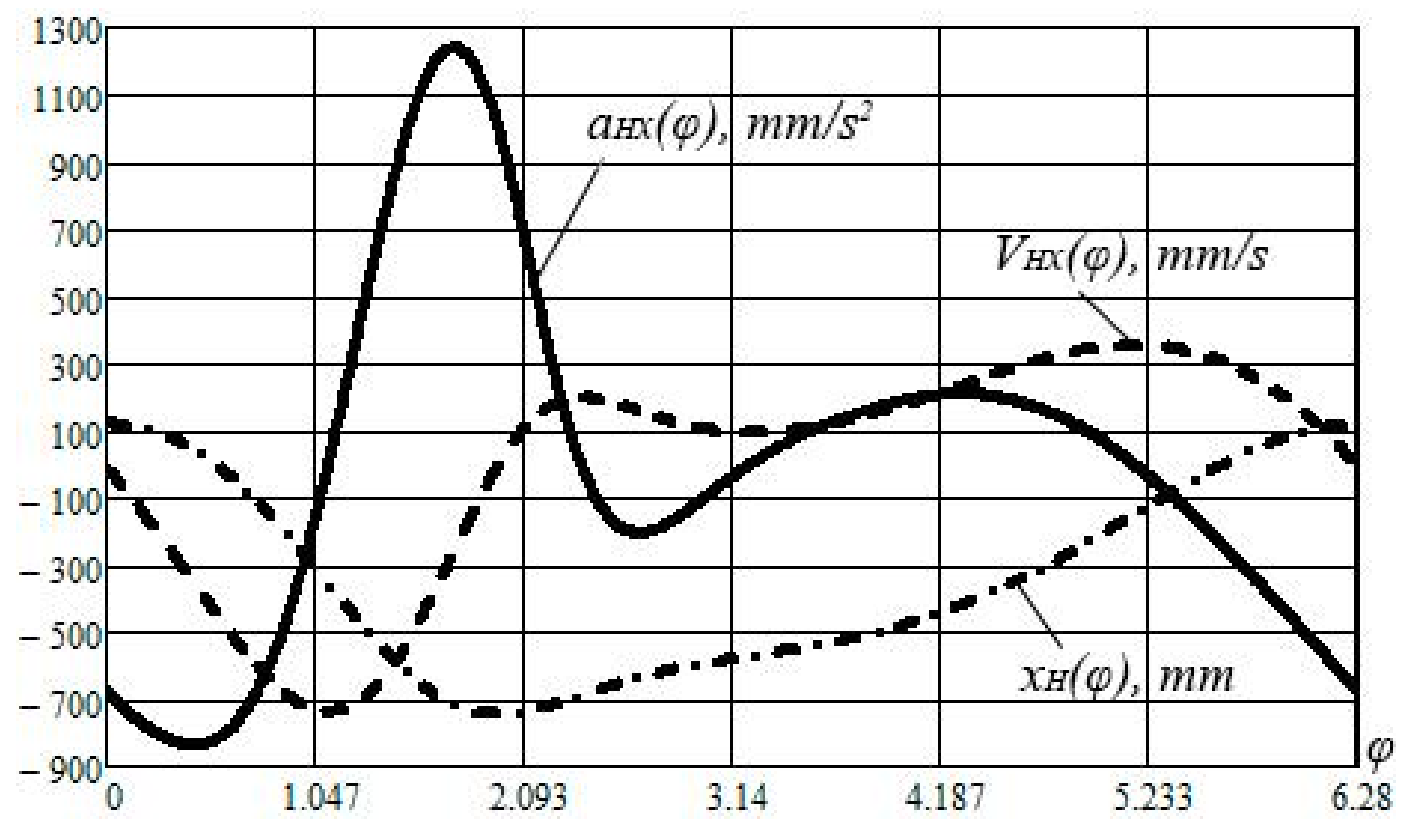

Fig. 6. Dependencies of horizontal displacement, speed and acceleration of the supporting foot of the walking mechanism on the driving crank rotation angle

Analysing the obtained graphical dependencies (Fig. 6), we may state that there are some periodic changes of horizontal speed and acceleration of the supporting point $H$. In particular, the speed $V_{H x}$ increases from $0.1 \mathrm{~m} / \mathrm{s}$ to $0.35 \mathrm{~m} / \mathrm{s}$ and then reduces to $0 \mathrm{~m} / \mathrm{s}$ during one step $\left(\varphi=180-360^{\circ}\right)$. Herewith, the acceleration increases from $0 \mathrm{~m} / \mathrm{s}^{2}$ to $0.2 \mathrm{~m} / \mathrm{s}^{2}$ and then reduces to $-0.7 \mathrm{~m} / \mathrm{s}^{2}$. This causes the necessity to ensure the equality of durations of both phases of the foot motion in order to prevent the simultaneous contact of several foots with the supporting surface. On the other hand, this problem may be solved by carrying out the further process of the walking mechanism parameters optimization [18].

Thus, the prospects of further investigations on the subject of the article may consist in optimization of geometrical parameters of the hinge-lever walking mechanism in order to ensure the zero-values of vertical speed and horizontal acceleration of the supporting point during the phase of its contact with the supporting surface by means of substantiation of the rational weight coefficients of the objective function and the expedient number of the synthesis input parameters.

\section{Virtual Experiments Performing and Simulation Modelling of the Supporting Foot Motion}

The solid-state model of experimental prototype of the walking mechanism (Fig. 7, a) has been designed using the SolidWorks software. This software is a system of computer-aided design, engineering analysis and manufacture preparation of any complexity and purpose. In the solid-state model, the plate $O B C$ conventionally represents the frame (body) of mobile robotic system. The driving crank is joined to the hinge $O$ and transmits the rotational motion to the hinges $S, M$ of the connecting rods $S D$ and $M G$, correspondingly. These rods are hingely joined to the rockers $C D L$ and $B G N$, which actuate the connecting rods $L K H$ and $N K$. The supporting hinge $H$ is used for joining the supporting foot; therefore, the kinematic characteristics of its motion will be the subject of further investigations and modelling in the system of engineering analysis SolidWorks Motion. It allows to carry out the complex 
dynamic and kinematic analysis of various mechanisms, as well as to perform their optimization according to the prescribed dynamic and kinematic criterions.

MapleSim software is a high-efficient system for modelling and simulation of various physical systems. It allows to compose various systems using specific functional blocks, among which there are special elements that model the behaviour of mechanical, hydraulic and electrical systems. For modelling the foot motions of the optimized walking mechanism, its simulation and functional diagrams were constructed in MapleSim software (Figs. 7, b, c). The module of generation of the constant angular velocity operates as the system driver and actuates the crank $O S(O M)$. The motion from the crank is transmitted to the hinge $H$ of the supporting foot joint using the system of rods and hinges presented in Figs. 7, $b, c$. The hinges $O, B, C$ are attached to the fixed pillar (walking machine body). Their coordinates $x_{B}, y_{B}, x_{C}, y_{C}$ were defined above as a result of optimization synthesis of the mechanism. Visual interpretation (simulation diagram) of the walking mechanism (Fig. 7,b) fully corresponds to its solid-state model designed in SolidWorks software (Fig. 7, a). The element Probe6 allows to construct the graphical dependencies of coordinates, speeds and accelerations of any point of the model being investigated, to which it is attached. In particular, we will analyse the motion of the hinge $H$ of the supporting foot attachment to the connecting rod $L K H$ (Fig. 7, c).

During the process of virtual experiments carrying out, we will analyse the kinematic characteristic of the supporting hinge $H$ motion within the period of one step. In particular, to a greater extent, we will consider the trajectory of the foot motion, its horizontal speed and acceleration during the phase of its contact with the supporting surface. Some results of modelling the motion of the walking mechanism obtained in SolidWorks software are presented in Fig, 8, a. The results of simulation modelling of the walking mechanism supporting foot motion in MapleSim software obtained with a help of the Probe 6 module are presented in Fig. 8, $b$.

On the basis of results of simulation modelling (Fig. 8), we may state that the supporting foot of the walking mechanism performs the maximal vertical displacement of $0.25 \mathrm{~m}$, and horizontal displacement of $0.9 \mathrm{~m}$. At the same time, the phase of the foot contact with the supporting surface fully ensures the prescribed step length $(700 \mathrm{~mm})$, and the phase of the foot transportation ensure the necessary height of the foot lifting $(200 \mathrm{~mm})$. The results of the virtual experiment fully corresponds to the theoretical trajectory of the foot (Fig. 5) constructed using the analytical dependencies (3)-(5) taking into account the parameters of the walking mechanism obtained on the basis of its kinematic synthesis.

Analysing the kinematic parameters of the supporting foot motion, in particular, the character of changing of its horizontal displacement, speed and acceleration (Fig. 8), we may state that during the phase of the foot contact with the supporting surface $\left(\varphi=180-360^{\circ}\right)$ the displacement of the point $H$ is not totally parallel to the $O x$ axis. This is confirmed by the fact that the speed $V_{H x}$ during one step $\left(\varphi=180-360^{\circ}\right)$ increases from $0.1 \mathrm{~m} / \mathrm{s}$ to $0.35 \mathrm{~m} / \mathrm{s}$, and then reduces to $0 \mathrm{~m} / \mathrm{s}$. Herewith, the horizontal acceleration increases from $0 \mathrm{~m} / \mathrm{s}^{2}$ to $0.2 \mathrm{~m} / \mathrm{s}^{2}$, and then reduces to $-0.7 \mathrm{~m} / \mathrm{s}^{2}$. Thus, we may state about the absolute convergence of the simulation experiment results (Fig. 8) and the results of theoretical modelling (Fig. 6) of the walking mechanism motion, as well as about the adequacy of the obtained analytical dependencies (3)-(5) for its kinematics analysing.

The prospects of further investigations should include the analysis of the influence of the optimization problems weight coefficients and the accuracy of choosing the initial estimates of geometrical parameters on the output parameters of the optimization synthesis. 


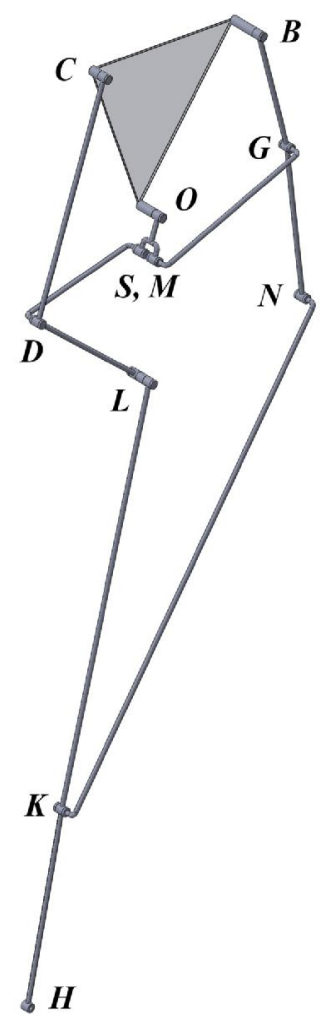

$a$

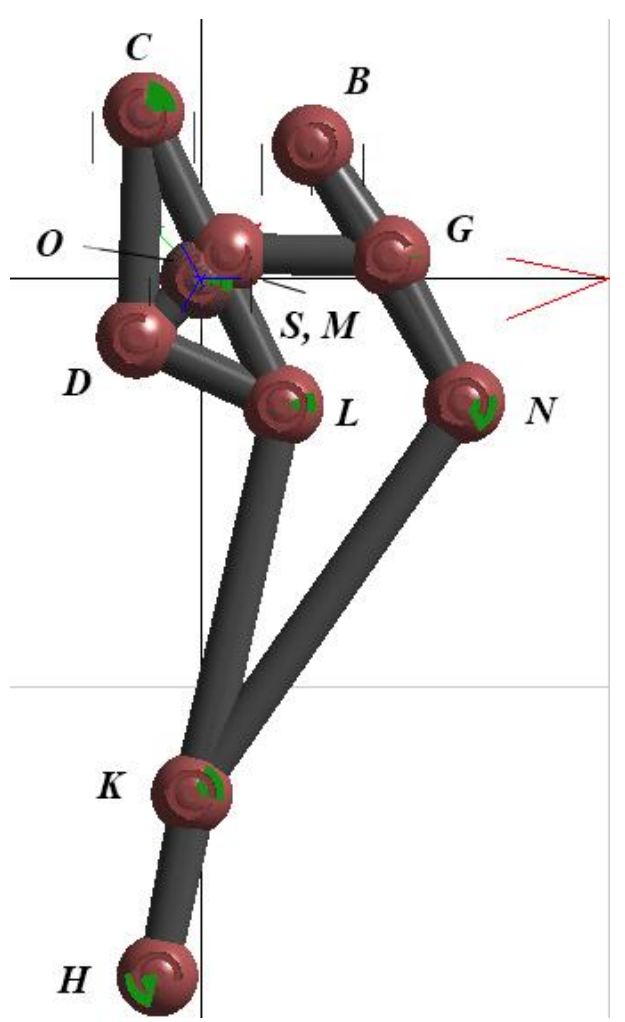

$b$

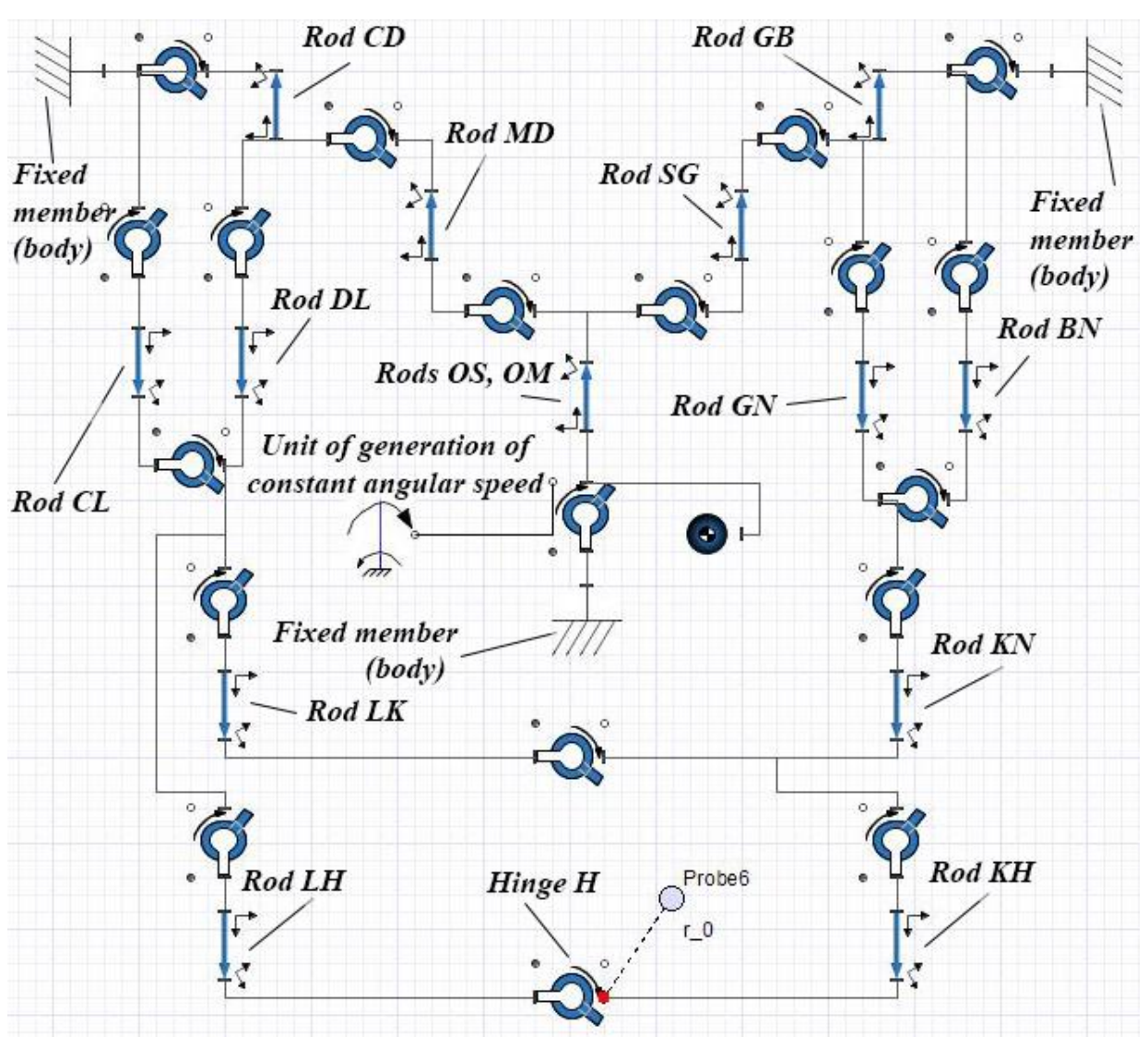

$c$

Fig. 7. Models developed for kinematic analysis of the walking mechanism: a-solid-state model designed in SolidWorks software; $c, b$ - functional and simulation models constructed in MapleSim software 


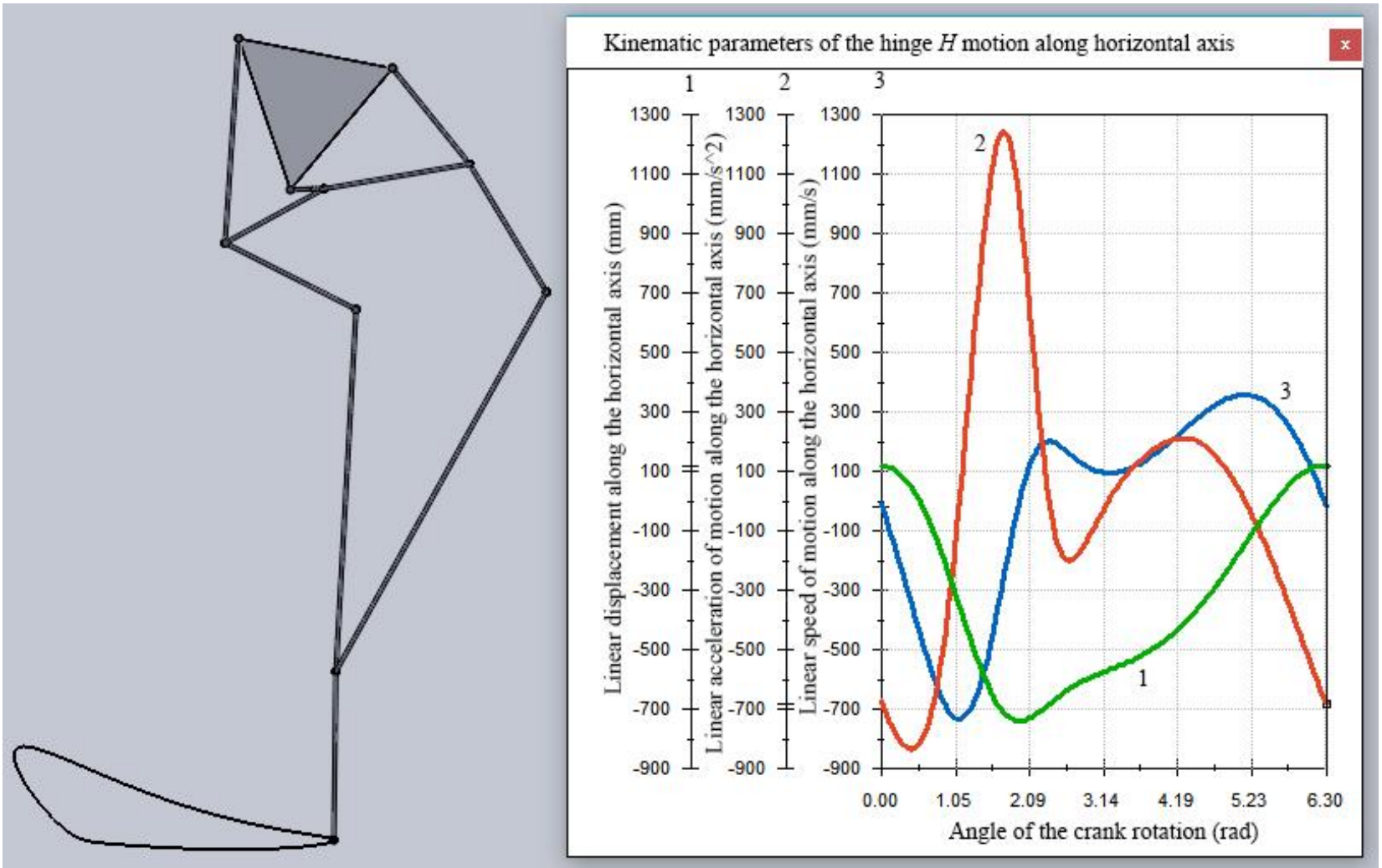

$a$
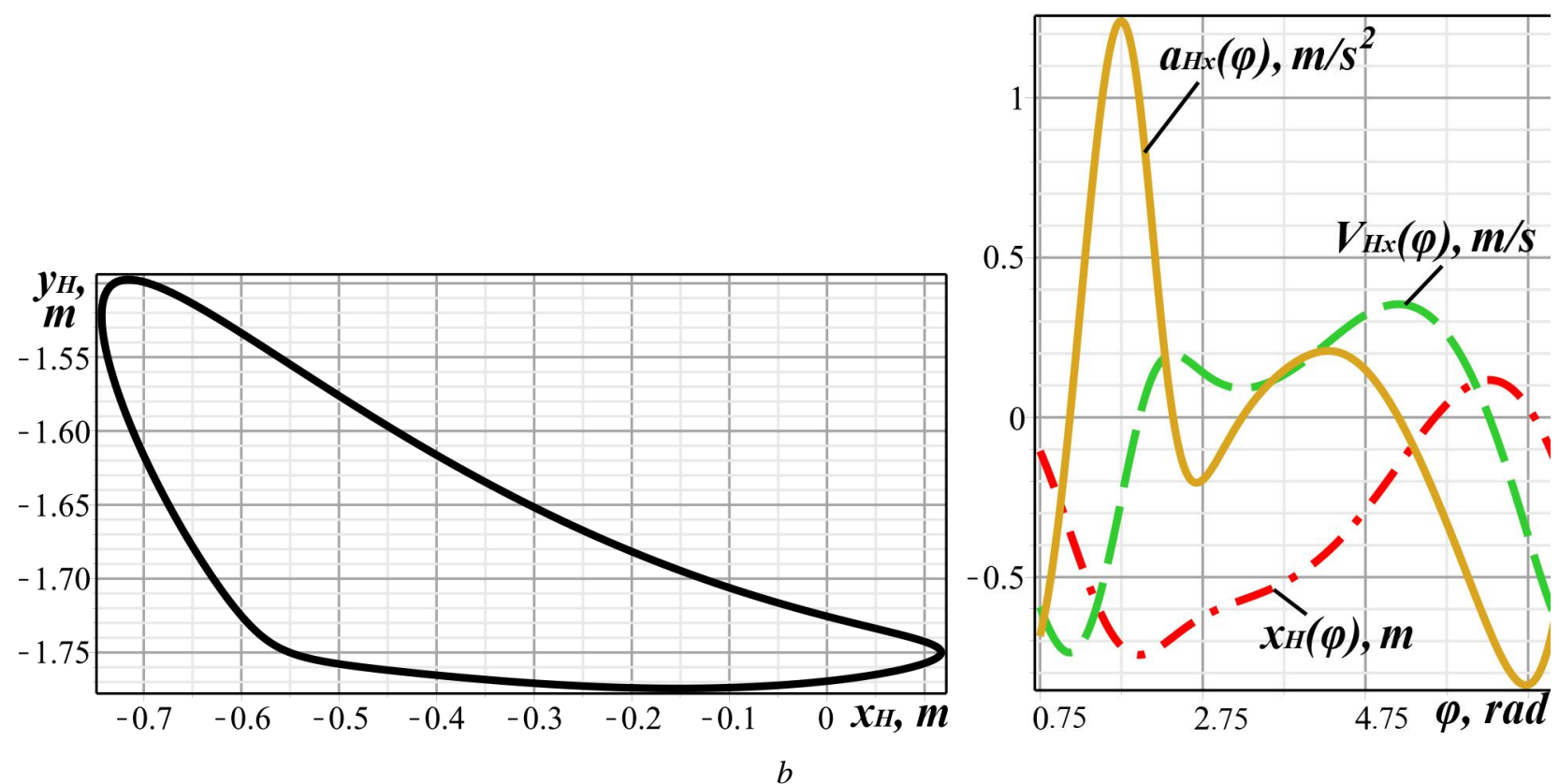

Fig. 8. Results of modelling the motion of the walking mechanism using SolidWorks (a) and MapleSim (b) software

\section{Conclusions}

In the paper, the structural synthesis of the hinge-lever walking mechanism base on three structural groups (Assur groups) of the $2^{\text {nd }}$ class $2^{\text {nd }}$ order 1 type and one driving link (the mechanism of the $1^{\text {st }}$ class) has been carried out.

The kinematic analysis of the synthesized mechanism has been performed and the analytical dependencies of the coordinates of the hinge of the supporting foot attachment as functions of the geometrical parameters of the mechanism and of the crank rotation angle have been derived. 
On the basis of the derived analytical dependencies, the corresponding function of the mechanism optimization has been proposed. After solving the optimization problem, the geometrical parameters of the walking mechanism have been defined.

Using the obtained geometrical parameters of the walking mechanism, the analysis of kinematic parameters of the supporting foot motion has been carried out on the basis of the derived analytical dependencies. The graphical interpretation of the supporting foot trajectory, speed and acceleration has been considered.

Using the SolidWorks and MapleSim software, the solid-state, functional and simulation models of the synthesized walking mechanism have been constructed. Performing the virtual experiments, the graphical dependencies of basic kinematic parameters of the supporting foot motion has been constructed.

Analysing the results of theoretical investigations and virtual experiments, the conclusion about their full confirmation (convergence) has been made. On the basis of the obtained results, the prospects of further investigations have been analysed.

\section{References}

[1] J. Billingsley, A. Visala, and M. Dunn, "Robotics in Agriculture and Forestry", in Springer handbook of robotics, B. Siciliano and O. Khatib, Eds. Berlin Heidelberg: Springer-Verlag, 2008, pp. 1065-1077.

[2] G. Carbone and M. Ceccarelli, "Legged Robotic Systemes", in Cutting Edge Robotics, V. Kordic, A. Lazinica, and M. Merdan, Eds. Rijeka, Croatia: InTech, 2005, pp. 553-576.

[3] E. Garcia, M. A. Jimenez, P. G. De Santos, and M. Armada, "The evolution of robotics research", IEEE Robot. Autom. Mag., vol. 14, no. 1, pp. 90-103, 2007.

[4] J. E. Shigley, The Mechanics of Walking Vehicles. Detroit, Michigan, 1960.

[5] V. Korendiy, "Analysis of Structure and Kinematics of Four-Bar Crank-Rocker Walking Mechanism", Ukr. J. Mech. Eng. Mater. Sci., vol. 1, no. 2, pp. 21-34, 2015.

[6] H. Funabashi, K. Ogana, Y. Gotoh, and F. Kojima, "Synthesis of Leg-Mechanisms of Biped Walking Machines (Part I. Synthesis of Ankle-Path-Generator)", Bull. JSME, vol. 28, no. 237, pp. 537-543, 1985.

[7] H. Funabashi, K. Ogana, I. Honda, and N. Iwatsuki, "Synthesis of Leg-Mechanism of Biped Walking Machines (Part II. Synthesis of Foot-Driving Mechanism)”, Bull. JSME, vol. 28, no. 237, pp. 544-549, 1985.

[8] P. A. Simionescu and I. Tempea, "Kinematic and Kinetostatic Simulation of a Leg Mechanism", in Proceedings of the 10th World Congress on the Theory of Machines and Mechanisms, 1999, pp. 572-577.

[9] W. B. Shieh and L. W. Tsai, "Design and Optimization of Planar Leg Mechanisms Featuring Symmetrical Foot-Point Paths", University of Maryland, 1996.

[10] G. V. P. Babu and N. A. N. Rao, "Design and Analysis of a Low Cost and Easy Operated Leg Mechanism for a Walking Robot", Int. J. Mech. Ind. Eng., vol. 2, no. 1, pp. 60-64, 2012.

[11] A. Aan and M. Heinloo, "Analysis and synthesis of the walking linkage of Theo Jansen with a flywheel", Agron. Res., vol. 12, no. 2, pp. 657-662, 2014.

[12] O. Al-Araidah, W. Batayneh, T. Darabseh, and S. M. BaniHani, "Conceptual design of a single DOF human-like eight-bar leg mechanism”, Jordan J. Mech. Ind. Eng., vol. 5, no. 4, pp. 285-289, 2011.

[13] S. Erkaya, "Trajectory optimization of a walking mechanism having revolute joints with clearance using ANFIS approach", Nonlinear Dyn., vol. 71, no. 1-2, pp. 75-91, 2013.

[14] F. Moldovan, V. Dolga, O. Ciontos, and C. Pop, "CAD design and analytical model of a twelve bar walking mechanism”, UPB Sci. Bull. Ser. D Mech. Eng., vol. 73, no. 2, pp. 35-48, 2011.

[15] S. Nansai, N. Rojas, M. R. Elara, and R. Sosa, "Exploration of adaptive gait patterns with a reconfigurable linkage mechanism", in Proceedings of IEEE International Conference on Intelligent Robots and Systems, pp. 4661-4668.

[16] V. M. Korendiy, O. S. Bushko, O. Yu. Kachur, and R. Yu. Skrypnyk, "Rozroblennia krokuiuchoho modulia na osnovi dvokh tsyklovykh rushiiv" ["Developing walking module based on two cycle engines"], Avtomatyzatsiia vyrobnychykh protsesiv u mashynobuduvanni ta pryladobuduvanni [Industrial Process Automation in Engineering and Instrumentation], vol. 49, pp. 26-35, 2015. [In Ukrainian].

[17] Ia. T. Kinytskyi, V. O. Kharzhevskyi, and M. V. Marchenko, Teoriia mekhanizmiv i mashyn $v$ systemi Mathcad [Theory of mechanisms and machines in Mathcad system]. Khmelnytskyi, Ukraine: RVTs KhNU Publ., 2014. [In Ukrainian].

[18] J. Collard, "Geometrical and Kinematic Optimization of Closed-Loop Multibody Systems", Université Catholique de Louvain, 2007. 